\title{
LA INVESTIGACIÓN ACCIÓN PARTICIPATIVA COMO HERRAMIENTA PARA LA TRANSFORMACIÓN INCLUSIVA DEL ESPACIO EDUCATIVO DE UNA ESCUELA DE EDUCACIÓN PRIMARIA EN ZARAGOZA
}

PARTICIPATORY ACTION RESEARCH AS A TOOL FOR AN INCLUSIVE TRANSFORMATION OF THE LEARNING SPACE OF A PRIMARY SCHOOL IN ZARAGOZA

\section{RECHERCHE-ACTION PARTICIPATIVE COMME OUTIL POUR LA TRANSFORMA- TION INCLUSIVE DE L'ESPACE ÉDUCATIF D'UNE ÉCOLE PRIMAIRE À SARAGOSSE}

\author{
Irene Ares Nicolás \\ Universidad de Zaragoza
}

\section{Resumen:}

En los últimos años, son muchos los centros y comunidades educativas que demandan la transformación de sus espacios consolidados aludiendo a la poca adecuación entre los espacios escolares y las metodologías de enseñanza y aprendizaje, las barreras arquitectónicas existentes, la falta de calidad estética de las escuelas o la carente diversidad (cultural, de género, funcional...) de las mismas, entre otras. Se plantea una investigación acción participativa en una escuela de Educación Primaria en la ciudad de Zaragoza, la cual desea iniciar un proceso de transformación, con el objetivo de entender cómo son y se usan los espacios educativos del centro, cuáles son las necesidades reales de la comunidad educativa y cómo debería llevarse a cabo una transformación que garantice dichas necesidades. En esta investigación de corte cualitativo se recogen de forma participativa las percepciones de los diferentes agentes implicados, mediante las técnicas de observación participante, grupos de discusión, entrevistas semi-estructuradas, actividades en el aula y un análisis arquitectónico del centro. Los datos recogidos han sido posteriormente analizados con el software NVIVO 12. Con los resultados obtenidos se ha llevado a cabo un diagnóstico colectivo del centro que servirá de punto de partida para la futura transformación de sus espacios.

Palabras clave: espacio educativo; arquitectura escolar; transformación socioeducativa; investigación acción participativa; inclusión. 


\begin{abstract}
Recently, schools and educative communities demand the necessity of transforming their consolidated spaces in reference to the inappropriate adequacy between spaces and learning methodologies, architectural barriers, the lack of aesthetic quality or the scarce diversity (cultural, gender-wise, functional...), among others. In this dissertation, a participatory action research is proposed, in a school that wishes to initiate a transformation process. This way, it arises the need of understanding how the spaces of this school are and how are they used, which are the needs of the agents of this educative community and how should a transformation responding to these necessities be. With the aim of answering to these questions, qualitative research has been proposed; considering participant observation, discuss groups with the agents of the educative community, activities that had been carried with students and an architectural analysis. A data analysis has been done through the software NVIVO 12. The obtained results of the analysis have helped to construct the participatory diagnosis which will serve as a beginning point for a future architectural transformation of the school.
\end{abstract}

Keywords: learning space; scholar architecture; socio-educational transformation; participatory action research; inclusion.

\title{
Résumé
}

Ces dernières années, de nombreux centres et communautés éducatives exigent la transformation de leurs espaces consolidés, en faisant allusion à la mauvaise adéquation entre les espaces scolaires et les méthodologies d'enseignement et d'apprentissage, les barrières architecturales existantes et au manque de qualité esthétique dans les écoles ou à leur manque de diversité (culturelle, de genre, fonctionnelle ...), entre autres. Une recherche-démarche participative est proposée dans une école primaire de la ville de Saragosse, qui veut entamer un processus de transformation. C'est ainsi que le besoin de comprendre comment sont les espaces éducatifs du centre et comment ils sont utilisés s'impose, quels sont les besoins des acteurs de la communauté educative et comment mener une transformation pour répondre à ces besoins. Cette recherche qualitative où les perceptions de tous les acteurs impliqués sont collectées de manière participative est proposée. À partir des techniques d'observation participante, des groupes de discussion, des entretiens semi-structurés, des activités en classe et d'une analyse architecturale du centre, les données ont été collectées puis analysées avec le logiciel NVIVO 12. Avec les résultats obtenus, un diagnostic du centre a été réalisé et il servira de point de départ à la future transformation de ses espaces.

Mots-clés: espace éducatif; architecture scolaire; transformation socio-éducative; recherche-démarche participative; inclusion. 


\section{INTRODUCCIÓN}

En el ámbito educativo, organismos internacionales como la OECD (2013) afirman que la mejora de la educación requiere, entre otros factores, de un cambio en la organización y una flexibilización del tiempo y del espacio en los centros educativos. Así, en los últimos años la temática de la arquitectura escolar y el espacio educativo ha adquirido un creciente interés en el panorama internacional de la investigación científica (Boddington y Boys, 2011; Cleveland y Fisher, 2014). En España se comparte esta tendencia por el estudio de la función didáctica del espacio, entendiendo su influencia en los procesos de enseñanza-aprendizaje y en las dinámicas convivenciales de la comunidad educativa (Bautista y Borges, 2013; Cela et al., 2016). Sin embargo, no existen muchas investigaciones que pongan atención al análisis de los procesos de transformación de espacios educativos ya consolidados en los centros escolares (Bazarra y Casanova, 2016).

En un contexto educativo caracterizado por la homogeneización en la enseñanza es necesario fomentar prácticas innovadoras e inclusivas que respondan a las necesidades de todo el alumnado (Vigo y Soriano, 2014). La escuela tiene el reto y la posibilidad de construir o transformar espacios que favorezcan procesos de enseñanza y aprendizaje para todo el mundo (Betrián et al., 2014). Este enfoque puede ser controvertido y difícil de entender, por lo que su desarrollo en ocasiones resulta complejo (Ainscow et al., 2006).

En este sentido, adquieren especial relevancia los llamados espacios colaborativos de aprendizaje entendidos como nuevos paradigmas educativos emergentes. Estos espacios, tanto formales como informales, como aquellos promovidos desde el eco-conectivismo, entendido como la manera de abordar el paradigma pedagógico conectivista en el aula, parten de la teoría de sistemas emergentes basados en las características de individuos y espacios en un momento dado (Barba-Guamán et al., 2018). Se trata de un modelo que busca crear comunidades conformadas por la cohesión de individuos, penalizando aquellas que no viven en comunidad. 
La presente investigación tiene como objetivo incidir en todas estas cuestiones, entendiendo la práctica de la transformación del espacio educativo como una herramienta que favorezca aspectos como la incorporación de nuevas metodologías pedagógicas, la inclusión en las aulas y la conformación de centros abiertos a la comunidad. Esta perspectiva otorga importancia a la creación de ambientes que motiven y permitan a la comunidad educativa sentirse a gusto para aprender y convivir.

\section{MÁRCO TEÓRICO}

Los seres humanos no siempre somos conscientes de la influencia que ejerce sobre nosotros el entorno en el que vivimos (Aragonés y Amérigo, 1998). El entorno se conforma por los diversos escenarios donde transcurre la vida de sus usuarios y los distintos significados individuales y compartidos que estos le confieren. Así, a través de este proceso interactivo, el espacio se convierte en ambiente (Aragonés y Amérigo, 1998; Kelz et al., 2015), entendido como todo aquello que rodea a las personas, desde un carácter social y físico (Staats, 2012). En el ámbito educativo, Pol y Morales (1986) definen ambiente como el conjunto de la edificación escolar, incluyendo espacios y equipamientos interiores y exteriores, emplazados en un contexto social y ambiental.

Este estudio entiende el espacio educativo como un elemento de integración donde valores como la sostenibilidad, la responsabilidad social o el compromiso e implicación con la diversidad generen un sentido de pertenencia que favorezca la inclusión del alumnado así como su propio proceso de aprendizaje (Hart, 2001; Novo, 2002). Aunque no existe un único modelo de buen espacio de aprendizaje (Boddington y Boys, 2011; Cela et al., 2016; Cleveland y Fisher, 2014), la mayoría de autores coinciden en otorgar un papel fundamental a la calidad ambiental del mismo.

En esta línea, Barrett y Zhang (2009) establecen una serie de elementos a tener en cuenta en el diseño arquitectónico: la estimulación que provoca el ambiente a partir de la configuración general del entorno; el color y las texturas; la sensación visual de orden y equilibrio; factores ambientales como la luz, el sonido, la tempe- 
ratura y la calidad del aire; así como la individualización, en relación a aspectos de elección y flexibilidad espacial. Los factores asociados al ambiente de la escuela proporcionan al niño un clima de seguridad (Viñao, 1993).

El factor estético, analizado como expresión de necesidades humanas es un elemento relevante en la transformación de espacios educativos (Aragonés y Amérigo, 1998). La estética cotidiana está relacionada con la experiencia del día a día y la apreciación del orden, la belleza, la limpieza o sus opuestos (Alonso-Sanz, 2017). Abarca desde aspectos decorativos hasta el diseño, configuración, tamaño y distribución del espacio escolar cumpliendo con las condiciones físicas necesarias para nuevos procesos de enseñanza, determinando la imagen que construimos y proyectamos de la institución escolar y configurando su propia identidad (Delgado, 2009; Errázuriz-Larraín, 2015).

El proyecto planteado de transformación tiene como objetivo intervenir en todos aquellos espacios detectados por la comunidad educativa que por un lado carecen de calidad ambiental y/o estética y por otro perjudican y/o no favorecen el proceso de enseñanza-aprendizaje, la inclusión o la convivencia en las aulas, entendiendo la convivencia escolar como el establecimiento de relaciones interpersonales en el contexto educativo con efectos positivos sobre el bienestar personal y que a su vez mitiguen comportamientos negativos como el bullying o acoso escolar, entre otros.

\section{MÉTODO}

\section{Diseño metodológico}

La investigación acción participativa en el ámbito educativo

La investigación acción participativa (IAP) tiene su origen en las aportaciones del sociólogo Kurt Lewin, surgiendo como una metodología que integra avances teóricos con transformaciones sociales, donde praxis y teoría se fusionan. Lewin (1946) planteaba un posicionamiento teórico y racional de los problemas a través de una secuencia de etapas, cada una de las cuales se compone de un proceso de planificación, acción y obtención de información sobre el resultado de esta acción, 
de manera que se retroalimentan una de otra. Se parte de la identificación y reconocimiento de un problema, la recogida de datos, la categorización de la información y la estructuración de las categorías, con el fin de lograr transformaciones en la vida social de la comunidad.

En el desarrollo de esta metodología destaca la figura de Fals Borda quien profundiza en la implicación del investigador en la comunidad, el análisis del conjunto de la misma, el desarrollo del nivel de conciencia de los miembros implicados y el desarrollo de grupos de acción. Introduce el concepto de investigación militante, caracterizada por su énfasis en la solución de problemas partiendo del compromiso con la comunidad o grupo (Fals, 1985).

En el área de la investigación educativa, la IAP pone especial énfasis en el desarrollo de procesos participativos donde el conjunto de la comunidad educativa se incorpora en el proceso para dar solución a los problemas planteados. Con un carácter sistemático y crítico se realiza una recolección pormenorizada de la información, la categorización y estructuración rigurosa de los datos cualitativos (Martínez, 2000). Su interés creciente se fundamenta en las posibilidades que ofrece, puesto que incluye la investigación pero también la educación y la acción (Balcazar, 2003).

Esta investigación surge de la confluencia de intereses de personas de distintos ámbitos profesionales que coinciden en compartir la necesidad de intervenir en el espacio físico de una escuela de Educación Primaria. Desde un enfoque interdisciplinar y comunitario se comparte el objetivo de generar procesos de transformación, no solo del espacio físico sino también de cambios en ámbitos como el educativo y el social.

El proyecto global contempla la secuencia de una serie de fases no lineales, en forma de espiral, que incluyen: el análisis de las principales necesidades, dificultades y potencialidades que presenta el centro y su comunidad educativa; el planteamiento de líneas de acción en todos los ámbitos, implicando al conjunto de la comunidad educativa; la movilización de recursos, la implementación de las actuaciones propuestas y la evaluación del proceso. La primera fase se consolida con la 
creación de un grupo motor o de acción, coordinado por una investigadora externa al centro. El investigador como agente externo apoya y facilita el proceso, favorece la creación de coaliciones, motiva la participación y permite la movilización de recursos (Balcazar et al., 1998).

Selener (1997) considera que el problema se origina en la comunidad y debe ser definido, analizado y resuelto por los participantes implicados. Por ello la IAP postula preguntas de investigación que son formuladas por los miembros de la comunidad junto a los investigadores externos, de manera que el diálogo conlleva el desarrollo de conciencia crítica en las personas participantes.

Siendo así, las preguntas de investigación del presente estudio surgen del diálogo entre la investigadora y parte de la comunidad educativa, principalmente del jefe de estudios del centro y parte del profesorado. Estas son: ¿cuáles son las necesidades de los agentes implicados: alumnado, profesorado, familias, equipo directivo...?, ¿cómo son y se usan los espacios educativos del centro?, ¿cómo implementar una transformación inclusiva que responda a esas necesidades?, ¿cómo implicar a la comunidad educativa?, ¿cuáles son las potencialidades y factores de riesgo? y ¿qué factores de índole física, educativa y social intervienen en un proyecto de estas características y cómo interactúan?

El diagnóstico participativo en el marco de la IAP

Una vez identificado el problema, la fase de diagnóstico adquiere especial relevancia ya que permite llevar a cabo el análisis de necesidades a la vez que facilita y motiva la implicación de los agentes de la comunidad educativa. Para ello esta fase se plantea con un hilo conductor que parte de identificar cuáles son las condiciones actuales y cuáles serían las condiciones deseables, así como las futuras.

En este proceso los miembros de la comunidad educativa concretan los problemas y necesidades, reflexionan sobre las potencialidades y las dificultades externas e internas y se implican directamente en la propia investigación-acción. La elaboración colectiva del diagnóstico permite desarrollar en los participantes un sentido de pertenencia al proceso de investigación, percibiéndolo como un esfuer- 
zo propio, que merece ser continuado (Selener, 1997).

\section{Contextualización de la investigación}

La escuela seleccionada es un centro público de una sola vía conformada por 9 unidades, desde $1^{\circ}$ de Educación Infantil (E.I.) hasta $6^{\circ}$ de Educación Primaria (E.P). El colegio se ubica en el Casco Histórico de Zaragoza, uno de los distritos más vulnerables de la ciudad. En él residen realidades multiculturales muy diversas, sin fuerte conflicto social pero con ciertas tensiones en la convivencia y predominio de relaciones de coexistencia, con fuertes desigualdades y altos niveles de pobreza y de segregación y estigmatización, a lo que se une una sensación generalizada de falta de espacios (PICH, 2013). Cabe reseñar que el centro es reconocido como Lugar de Memoria Histórica al localizarse en un antiguo palacio sede del Tribunal de la Inquisición convertido en prisión habilitada para mujeres durante la posguerra. La estética carcelaria unida al mal estado de sus instalaciones conlleva una falta de calidad ambiental y estética escolar, notable por el conjunto de la comunidad educativa y del barrio.

\section{Participantes}

El diseño de la fase de diagnóstico asume la participación e implicación de toda comunidad educativa, profesionales del ámbito social y una arquitecta encargada de la coordinación de la presente investigación.

El profesorado participante fue la totalidad del claustro, el director, el jefe de estudios y la responsable de las actividades extra-escolares. En total, 19 maestros y maestras, de los cuales 12 eran mujeres y 7 hombres. Además, la voz del alumnado se planteó como fundamental, por lo que se contó con la participación del total de alumnos y alumnas matriculados en el centro durante el curso 2018-2019, en total 164 entre las etapas de E.I. y E.P. También participaron 22 madres, padres o tutores legales, junto con dos trabajadoras sociales, de una entidad social del barrio y del área de Servicios Sociales del Ayuntamiento de Zaragoza.

\section{Procedimiento de recogida de datos}

En la recogida de datos, atendiendo a las características particulares de los y 
las participantes, así como a los núcleos de interés, se utilizaron varias técnicas cualitativas y participativas: observación participante, entrevistas semi-estructuradas, grupos de discusión, actividades de aula y grupos interactivos con el alumnado, una evaluación técnica del espacio, así como la participación en jornadas, revisión de documentación y de experiencias implementadas en el territorio aragonés.

La implicación del profesorado y de la dirección del centro era fundamental, por lo que se llevaron a cabo dos entrevistas semi-estructuradas iniciales con el jefe de estudios y posteriormente frecuentes conversaciones informales, recogidas a través de grabaciones y anotaciones en un cuaderno de campo. En dichas entrevistas se elaboró previamente un guión de preguntas que trataban de esclarecer desde el punto de vista del equipo directivo cuál era la situación de la escuela a la hora de iniciar el proceso, las dificultades técnicas, económicas, personales,...que según su criterio lo dificultarían, sus preocupaciones y expectativas con el proyecto. La investigadora responsable del presente estudio fue la encargada de llevarlas a cabo. Debido a que no se pudieron contrastar los datos con otro investigador, fueron contrastados posteriormente con los obtenidos en los grupos de discusión del profesorado.

Así, se realizaron dos grupos de discusión, uno con el profesorado de E.P. (quedará reflejado como G.P. a partir de este momento) y otro con las maestras de E.I. (G.I.). El número de participantes fue de 11 y 4, respectivamente, participando el total del claustro de la escuela. La inexistencia de Asociación de Madres y Padres de Alumnos (AMYPA) requirió de la colaboración del profesorado para llevar a cabo una muestra selectiva de 19 madres y 3 padres, con los cuales se realizaron dos grupos de discusión de 9 y 13 participantes. Los criterios de selección de los y las participantes fueron la disposición de las mismos por participar y su voluntad por formar parte del proyecto, el cual se había explicado previamente; así como el conocimiento del idioma. Por último, se realizaron dos entrevistas semi-estructuradas a las dos trabajadoras sociales, con el fin de conocer la realidad del barrio y los recursos que podía disponer el centro en este proceso. 
Las preguntas planteadas en los grupos de discusión, estaban basadas en la fundamentación realizada a nivel científico y el análisis técnico del centro. Tenían como objetivo detectar las principales necesidades y dificultades del mismo, establecer una jerarquización e interacción de las mismas, así como avanzar en posibles soluciones y alternativas. Se realizaron grabaciones de todas ellas para su posterior análisis.

La recogida de información con el alumnado se llevó a cabo a través de observación participante y actividades de aula, entre los meses de abril y junio de 2019. Las actividades de aula se adecuaron a la edad de los y las alumnas. En el caso de E.I. y primer ciclo de E.P. se realizó un cuentacuentos y se utilizó una técnica indirecta como es el dibujo para que los niños y niñas plasmaran las cuestiones planteadas. Con el resto del alumnado de E.P. se aplicó una técnica participativa emulando un juicio en el aula en el cual los alumnos y alumnas debían reflexionar y debatir sobre los espacios del centro y su transformación. Este proceso culminó con una sesión de grupos interactivos en la que, a través de distintas técnicas artísticas, el alumnado reflejó sus propias propuestas. Los dibujos y materiales generados fueron recopilados para la posterior recogida de datos, así como las grabaciones de las sesiones y las anotaciones en el cuaderno de campo de la investigadora.

La arquitecta y coordinadora del proyecto fue la encargada de llevar a cabo el análisis técnico y arquitectónico, así como la recogida de todos los datos transcritos para su posterior análisis.

\section{Procedimiento de análisis de datos}

El análisis de datos se realizó con el programa informático NVIVO 12. Siguiendo a Beach \& Dovemark (2007), se indagó en varios núcleos de interés surgidos del estudio del estado de la cuestión del objeto de investigación y el diálogo con la comunidad educativa. En concreto estos fueron los déficits espaciales y de calidad ambiental; la convivencia u socialización; la diversidad e inclusión en el centro; y las metodologías de enseñanza utilizadas por el profesorado.

En un primer acercamiento al estudio, la arquitecta e investigadora realizó 
una evaluación de aspectos técnicos, organización y uso de los diferentes espacios del centro; seguido de un análisis con el software estadístico de los datos recogidos desde los núcleos mencionados, con la finalidad de observar cómo se interrelacionaban ambos análisis.

Así emergieron nuevas categorías, principalmente aquellas referidas al propio proceso participativo, por lo que posteriormente se tomó la decisión de organizar los datos en tres dimensiones o meta-categorías: la dimensión física; la dimensión educativa y social; y el proceso metodológico, para así concretar las categorías de las tres dimensiones (Tabla 1) y sus interrelaciones. La concreción de categorías y núcleos de interés, así como el uso de NVIVO 12 permitió la aproximación a la complejidad del entorno socio-educativo.

\section{Tabla 1}

Meta-categorías y categorías

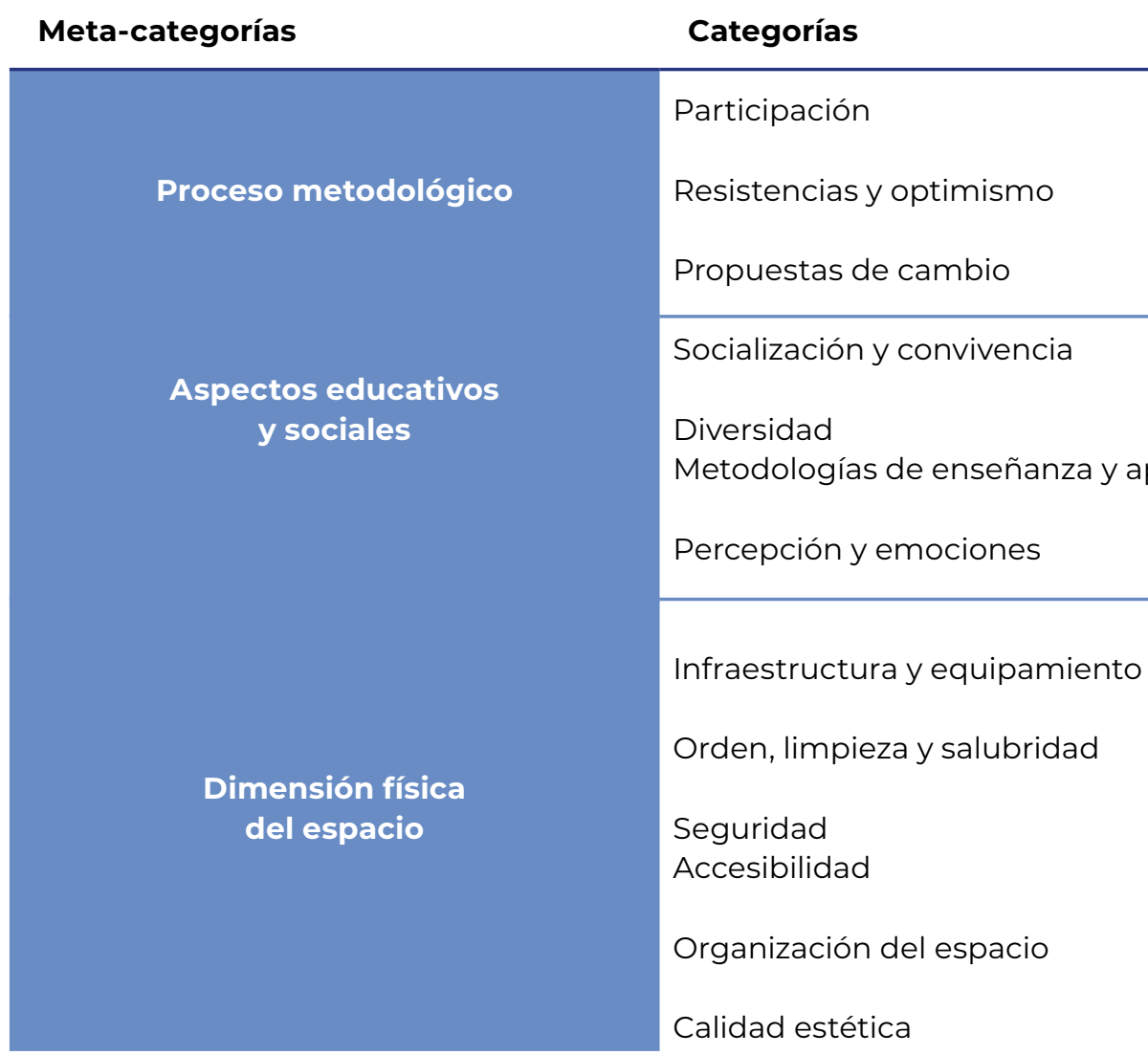

Nota. Fuente: Elaboración propia. 
Tal y como se ha comentado, en el transcurso del análisis, la naturaleza de la metodología de investigación reveló que muchos de los datos no pertenecían a la dimensión física del espacio, ni a aquellos aspectos relativos a la dinámica relacional del centro, sino al propio proceso de la investigación acción participativa. En esta meta-categoría se incluyó toda la información relativa a la participación de la comunidad educativa, considerando la participación como una categoría en sí misma, al ser un factor imprescindible en el proyecto de transformación y en la propia investigación. Las resistencias y optimismo de la comunidad se revelaron también como categoría, puesto que determinarían las fortalezas y debilidades del proyecto. Por último, el desarrollo del diagnóstico participativo dió pie a que el equipo directivo, el alumnado, el profesorado y las familias formularan sus propuestas y deseos para el proyecto, aportando información sobre sus necesidades reales, constituyendo una categoría que deberá tenerse en cuenta en la siguiente fase del proyecto: el diseño del mismo.

Por su parte, los aspectos educativos y sociales constituyen una meta-categoría y engloban todos aquellos referidos a las personas que habitan el espacio y las relaciones interpersonales y con el propio espacio, incluyendo la socialización y convivencia, la diversidad, las metodologías de enseñanza-aprendizaje puesto que determinan la forma de relacionarse en el centro, y la percepción y emociones que el espacio producía en los y las participantes.

Por último, la meta-categoría de la dimensión física del espacio surge del estudio del marco teórico y el análisis técnico realizado por la arquitecta investigadora, contrastando los datos con los obtenidos en los grupos de discusión y entrevistas. Estas categorías (infraestructura y equipamiento; orden, limpieza y salubridad; seguridad; accesibilidad; organización del espacio y calidad estética) hacen referencia a todos los aspectos físicos y técnicos que determinarán la definición del diseño del proyecto de transformación.

Una vez analizados los datos, el procedimiento de triangulación se llevó a cabo mediante el contraste de técnicas, así como de la información obtenida de 
los diferentes grupos de discusión y las entrevistas, es decir, entre todos los agentes implicados. Además, una vez extraídos los resultados se realizó un contraste de los mismos con dos investigadores externos al estudio, así como su devolución a la comunidad educativa con el fin de modificar o detallar aquello que no fuera pertinente o significativo. La triangulación metodológica como estrategia de investigación tenía como objetivo asegurar la validez y la confiabilidad de la información obtenida.

Por último, la investigadora principal junto con una de las trabajadoras sociales participantes del proyecto, realizaron un análisis DAFO, entendido como instrumento no meramente descriptivo sino como una herramienta estratégica válida para la elaboración del diagnóstico (Aliaga et al., 2018). Este análisis permitió sintetizar la información más significativa y plantear las líneas de intervención a futuro.

\section{RESULTADOS}

\section{Resultados sobre el proceso metodológico}

Proceso participativo

Al inicio de la investigación, cabe reseñar que la poca participación de las familias en el centro se valoraba por parte del profesorado y el equipo directivo como un obstáculo para la misma pero también como un reto a lograr en el desarrollo del proyecto. La movilización de toda la comunidad educativa se percibía como un elemento que impulsaría el proceso de transformación. Además, se constataba una cierta predisposición institucional a crear un centro educativo abierto al barrio y romper así con la estigmatización percibida del colegio: "Si tenemos como objetivo acercar a las familias a la escuela, plantearles un proyecto bonito y pedirles ayuda puede ser una buena forma para acercarnos." (Participante 1 del G.P.1)

Durante la recogida de datos, los niños y niñas mostraron su agradecimiento por el hecho de que se les escuchara y pidiera opinión sobre sus necesidades, lo que motivó al grupo motor de la investigación a continuar con la misma.

Resistencias y optimismo ante el cambio: dos caras de una misma moneda

En un primer momento, se percibió un cierto pesimismo por parte del profesorado a la hora de afrontar un proceso de transformación de espacios, principal- 
mente debido a la falta de tiempo y de financiación. Las propias dificultades estructurales y económicas, junto al sentimiento de abandono y soledad debido a la poca implicación institucional y la estigmatización que sufría el centro, condujeron un discurso de frustración que no permitía ver cómo iniciar actuaciones a nivel micro o impulsar acciones a un nivel macro: "Yo el problema lo veo en el apoyo de la DGA. Nos tienen considerados un colegio ghetto y pueden decir "si han estado así tanto tiempo, déjalos como están". (Participante 2 del G.P. 2)

Sin embargo, la creación del grupo motor y el propio proceso de elaboración del diagnóstico fueron valorados como un incentivo al optimismo y al espíritu colaborador, percibiéndose la posibilidad de que "era posible".

Propuestas de cambio en las distintas dimensiones

Durante la recogida de datos, se realizaron diversas propuestas por parte de la comunidad educativa. Para el profesorado la propuesta más aceptada implicaba un cambio en la organización general del centro, así como la creación de aulas temáticas adaptadas a la especificidad de cada material, lo que suponía también un cambio en el modelo pedagógico: "Podríamos crear espacios grandes donde se pueda dar clase. En la biblioteca hay un espacio donde se puede leer, ¿Por qué no se puede dar lengua ahí? (...) Que cada aula tenga una temática..." (G.P. 1)

Por su parte, el alumnado reclamaba diversidad de actividades y juegos en los espacios. Se percibía una voluntad por entender todos los espacios del centro como lugares de aprendizaje, no únicamente las aulas: "Que hubiera grupos de gramática, de teatro, una zona de cine..." (Alumno de $5^{\circ}$ E.P.), "En la biblioteca poder jugar al escondite, al halflife en el ordenador, leer quien quiera y jugar a muchos juegos. (Alumna de $6^{\circ}$ E.P.)

Cabe reseñar que algunas de las aportaciones buscaban mejorar la convivencia y las relaciones interpersonales entre los diferentes agentes de la comunidad educativa, lo que evidenciaba esta necesidad recogida en el propio diagnóstico: "Hay que generar espacios de convivencia, rincones de lectura, en los pasillos..." (G.P. 10), "Yo propongo espacios de convivencia que se puedan utilizar en el recreo, para 
estar con familias, tertulias dialógicas...Hacer espacios en los que se pueda estar." (G.P. 9)

\section{Resultados referentes a aspectos educativos y sociales}

Socialización y convivencia

Una de las categorías analizadas fue el papel del espacio en las dinámicas relacionales y de convivencia de la escuela. Profesorado y alumnado coincidían en que la convivencia era uno de los principales problemas, especialmente entre los alumnos y alumnas más mayores. Los espacios que primero se identificaron con la convivencia y los procesos de socialización fueron aquellos dedicados al tiempo de recreo.

En el centro estos espacios no eran los mismos para la etapa de infantil y la de primaria. La falta de espacio en el patio exterior conllevaba que el patio de infantil se ubicara en el hall interior, un espacio de tránsito no habilitado para albergar un patio infantil. Esta situación no favorecía la convivencia ni la interacción entre el alumnado de ambas etapas, ya que no existía un espacio compartido. Desde el principio, el profesorado evidenciaba el componente visual, la importancia de poder verse para poder interactuar; sin contacto no hay posibilidad de interacción, de compartir experiencias: "El espacio no permite que nos veamos todos, hay un ala (infantil) y otra ala (primaria) y no hay un espacio compartido." (G.P. 3)

Sin embargo, los propios alumnos y alumnas eran conscientes de que la interacción no está exenta de dificultades. Eran los espacios de intercambio: patio, hall, escaleras... donde generalmente se producían en mayor medida los conflictos. Cuando se habla de convivencia, el espacio trasciende a lo meramente físico y se entienden los espacios de convivencia como zonas dentro de otras que facilitan o posibilitan el contacto e interacción. El colegio entendido como un espacio abierto, de barrio y del conjunto de la comunidad educativa se visualizaba cuando las madres demandaban espacios propios dentro del centro donde poder juntarse y realizar actividades, espacios que permitieran su participación e incluso la reivindicación de un centro más adecuado a las necesidades de sus hijos e hijas. 


\section{La diversidad como factor espacial}

No siempre se ha analizado la relación entre aspectos como la diversidad en sus diferentes dimensiones y el espacio en cual se producen las interacciones sociales. En este estudio se tuvo en cuenta la interculturalidad, el género y la diversidad funcional.

La diversidad cultural es una de las características más relevantes del colegio, el porcentaje de alumnado que procede de otros países y de minorías étnicas es muy alto (alrededor de un 95\%), lo que convierte al centro en un espacio intercultural en sí mismo, con diferentes visiones del espacio. Por otro lado, la atención a la diversidad funcional se veía altamente limitada por las barreras arquitectónicas que presentaba el colegio. El centro no estaba adaptado para personas con diversas necesidades funcionales, comunicativas o intelectuales.

A todo ello había que añadir que la dimensión y configuración de las aulas no permitía que se pudiera disponer un espacio para el apoyo dentro de la propia aula de referencia del alumnado, lo que dificultaba la inclusión en las aulas. Además, la diferencia de uso del espacio en función del género se evidenciaba especialmente en el patio de recreo puesto que la disposición del mismo no permitía que existiera diversidad de juego; fueron las propias alumnas quienes manifestaron que no sentían como propio ese espacio.

\section{Espacio y metodologías de aprendizaje}

Este centro constituye una comunidad de aprendizaje en la cual voluntarios, vecinos y vecinas del barrio participan activamente en la vida del centro, principalmente en las sesiones de grupos interactivos que todos los cursos realizan varias veces a la semana, así como en tertulias dialógicas. Estas metodologías se intercalan con las sesiones habituales de clase magistral. Sin embargo, no se disponía de un espacio flexible y habilitado para poder llevar a cabo estas metodologías: “¿Hacemos grupos interactivos? Pues tener un espacio preparado con mesas para ello. Pero habría que quitarnos de la cabeza que este es nuestra aula y de aquí no salimos. El cambio de metodología es importante." (G.P. 1) 
A lo largo de la fase de diagnóstico se planteó entender la transformación de espacios como un proceso ligado al cambio metodológico en el centro, lo que supuso un punto de inflexión en el transcurso de la investigación. El claustro reflexionó sobre el tiempo que sus alumnos y alumnas pasaban dentro del aula de referencia y propusieron continuar introduciendo metodologías más activas, utilizando el espacio de forma más flexible y abierta.

Percepción y emociones en relación al espacio

Sabemos que un espacio genera emociones y sentimientos en las personas que habitan en él. Esto explica que el alumnado manifestara su preferencia por espacios con más luz o amplitud, puesto que en ellos se sentían mejor y más alegres. Además, no se debe olvidar el significado subjetivo que adquiere el color. Fueron varios los alumnos y alumnas que expresaron que el tono rojo apagado del patio les recordaba a la sangre y les evocaba sentimientos negativos: "No me gusta el color rojo en el patio, me recuerda a la sangre de las guerras" (alumno de $3^{\circ}$ E.P.)

Por otro lado, algunas familias, fundamentalmente las de etnia gitana, manifestaron un arraigo al centro y al barrio. Esto contrastaba con la percepción del profesorado, que consideraba que factores como la continua incorporación de nuevas familias, la mayoría de países de diferente procedencia, junto a la alta movilidad del alumnado a otros centros, problemas de convivencia, entre otras causas, influían en la falta de sentimiento de pertenencia del alumnado y sus familias.

\section{Resultados referentes a la dimensión física del espacio}

Infraestructura y equipamiento

El conjunto del claustro y el equipo directivo coincidía en enunciar y denunciar el mal acondicionamiento térmico y acústico del colegio. Este hecho, causado principalmente por la mala orientación del edificio, así como su antigüedad y el inadecuado estado de los cerramientos, conllevaba graves problemas que dificultaban el seguimiento de las clases y la convivencia en el colegio: "En verano es horroroso, es imposible estar en las aulas. Ha habido años que he tenido que sacar a todos los chiquillos e irnos a otro aula." (G.P. 5). "Hay tanto ruido (en el hall interior) 
que si te vienen a contar un problema no se les escucha..." (G.I. 1)

El mal estado de las persianas y la falta de cortinas en la mayoría de las aulas no permitía utilizar materiales digitales proyectados durante las sesiones, así como generar zonas de sombra en los meses de más calor. Otra de las prioridades para las maestras de E.I. era la falta de tomas de agua y baños en sus aulas. El aula de plástica tampoco disponía de toma de agua por lo que los alumnos y alumnas debían salir a los baños comunes del pasillo para limpiar los utensilios utilizados. Además, el profesorado y el alumnado resaltaban el mal estado de los baños, calificados como viejos e insalubres. Esta circunstancia era especialmente importante ya que muchos de los niños se duchan en el colegio después de la clase de Educación Física.

Orden, limpieza y salubridad

Toda la comunidad educativa resaltaba un problema general de limpieza y acondicionamiento de espacios. Las aulas no disponían de espacio suficiente para el almacenaje, estaban abarrotadas de material y mobiliario en desuso. Además, existían espacios en el centro que servían únicamente para almacenaje de material inservible: "Dentro del aula hay mucho mobiliario que no se utiliza. Habría que organizar la clase y tirar lo que no sirve." (alumna de $6^{\circ}$ E.P.)

Por otro lado, el mal estado del sistema de fontanería del centro conllevaba estancamientos de agua causando problemas de salubridad y mal olor en algunas zonas del colegio. A su vez, las filtraciones de agua debidas al mal estado de los cerramientos provocaban problemas de humedad tanto en el exterior como en el interior del edificio, lo que sin duda afectaba negativamente a la calidad ambiental del centro escolar.

\section{Seguridad}

El profesorado y las familias destacaron desde su preocupación por la seguridad de los alumnos y alumnas, especialmente en los accesos y en el patio exterior, al tratarse de un espacio reducido con muy poca separación entre pilares donde los niños corrían y jugaban pudiendo resultar peligroso para ellos. Además, el acabado del pavimento con pintura para carreteras no permitía que se absorbiera el agua 
de lluvia, provocando continuos resbalones cuando el alumnado jugaba o realizaba las actividades de Educación Física: “Y el patio para Educación Física. La pintura del suelo resbala en el momento que llueve. Es peligrosísimo" (G.P. 6).

El hecho de que el mobiliario y el equipamiento estuvieran en mal estado implicaba también un problema de seguridad que condicionaba el día a día en las aulas para el profesorado y el alumnado.

\section{Accesibilidad}

Las aulas de E.I. no estaban adaptadas para esta etapa educativa y no disponían de baños en su propio aula, ni en su mismo nivel; así, los niños y niñas de infantil debían subir y bajar escaleras para ir al baño. El hecho de que tuvieran que ir acompañados dificultaba la labor de las maestras en su día a día en el aula: "Que los baños estén bajando las escaleras dificulta la clase porque te pasas el día yendo y viendo porque todavía no controlan lo de subir y bajar escaleras" (G.I. 3).

Es importante resaltar la falta de adecuación del centro para personas con movilidad reducida. El ala central del edificio cuenta con ascensor; sin embargo, a las aulas en las alas laterales se accedía a través de escaleras lo que impedía el acceso para alumnos y alumnas en silla de ruedas. Además, la entrada al edificio desde el patio exterior de recreo - acceso habitual de los niños al centro - tampoco era accesible en este sentido.

\section{Organización del espacio}

La falta de espacio y las malas condiciones térmicas y acústicas en las aulas dificultaban el seguimiento de las clases y acarreaban problemas de convivencia. Las asignaturas específicas no contaban con un espacio propio adaptado y preparado para sus necesidades particulares. A su vez, existían espacios con mejores condiciones ambientales que no estaban lo suficientemente habilitados para la labor docente, por lo que no se aprovechaban o se utilizaban escasamente.

En contraposición a la falta de espacio en las aulas, uno de los atractivos del colegio son los grandes y luminosos espacios intermedios del mismo: el hall interior y los pasillos de las plantas superiores. Sin embargo, estos espacios prácticamente 
no se utilizaban ni estaban adaptados para albergar otro tipo de usos de carácter relacional.

Respecto al patio exterior, profesorado y alumnado coincidía que el problema era la falta de espacio, que como hemos comentado repercutía negativamente en la convivencia en el recreo. El campo de fútbol ocupaba la totalidad del patio por lo que no existía espacio para otros juegos, descansar, almorzar o hablar. Esta falta de diversidad conllevaba que el alumnado que no quería jugar a fútbol expresara su descontento por no disponer de un espacio para otras actividades: "A los alumnos de infantil no les gusta salir fuera porque fuera no tienen nada para poder hacer. (G.I. 1), "Voy a dibujar el patio con un espacio para nosotras." (alumna de $3^{\circ}$ E.P.).

Se destacó también la inexistencia de un espacio donde las familias se pudieran reunir con el claustro, con el equipo directivo o entre ellas lo que, tal y como señalaban, dificultaba su participación en el centro, así como la relación entre las familias y la escuela.

\section{Calidad estética}

Toda la comunidad educativa coincidía en que la estética del centro era más carcelaria que escolar. El edificio, de estilo brutalista, albergó durante la segunda mitad del siglo XX una cárcel de mujeres y no se había conseguido romper con esa imagen. El hormigón visto, la tonalidad gris de la fachada, las rejas en las ventanas... eran algunos de los elementos que contribuían a ello: "Las rejas en las ventanas hacen que el cole parezca una cárcel" (alumno de $5^{\circ}$ E.P.), "Que el patio de infantil, parezca un patio de infantil" (G.I. 3).

En el interior del edificio, especialmente en la planta baja, la tonalidad de las paredes era muy oscura lo que generaba una sensación todavía menos acogedora.

\section{Resultados del análisis DAFO}

Este método posibilita construir estrategias de mejora partiendo del análisis confrontado de factores internos y externos (Tabla 2). Los factores internos pueden ser atributos positivos (fortalezas) o negativos (debilidades). Los factores externos analizan elementos independientes al objeto valorado que podrían influir positiva- 
mente (oportunidades) o negativamente (amenazas) (Wang et al., 2014).

Tabla 2

Análisis DAFO

DEBILIDADES

¿Qué hay que transformar?
AMENAZAS

¿Cuáles son los obstáculos?
- Mal acondicionamiento térmico y acústico.

- Problemas de seguridad, accesibilidad y salubridad.

- Falta de organización de espacios.

- Estética carcelaria.

- Equipamiento y mobiliario en mal estado.

- Problemas de convivencia.

- Falta de interacción entre infantil y primaria.

- Falta de diversidad de juego y actividades
OPORTUNIDADES

¿Qué aspectos del centro favorecen

la consecución de nuestros objetivos?
Pesimismo ante las graves deficiencias. Falta de implicación de las administraciones públicas.

- $\quad$ Falta de tiempo por parte del profesorado.

- Falta de presupuesto propio.

- Escasa participación de las familias e inexistencia de AMYPA.

Estigmatización del centro y del distrito.
- Colegio referente en el barrio y la ciudad.

- Condiciones espaciales con posibilidades de cambio.

- Equipo interdisciplinar.

- Figura coordinadora del proyecto.

- Proceso sujeto a una investigación científica.

- Implicación del equipo directivo.

- Centro constituido como comunidad de aprendizaje.

- Motivación del profesorado y formación en metodologías activas y participativas.
Red social en el barrio.

Participación de instituciones públicas (Zaragoza activa y Plan Integral del Casco Histórico).

- Participación de colectivos de otras disciplinas: artísticas, sociales..

- Apertura e integración del centro educativo en el barrio.

\section{Discusión y conclusiones}

En la presente investigación se indagó en las necesidades del centro en re-

lación al espacio de su dimensión física, social y educativa, así como los aspectos derivados del propio proceso metodológico de la IAP.

Otros procesos de transformación de espacios educativos trabajaron con las posibilidades materiales, espaciales, visuales, sonoras y lumínicas, entre otras, que nos ofrece el ambiente (Errázuriz-Larraín, 2015) con el objetivo de mejorar su calidad estética entendiendo esta como expresión de necesidades humanas (Aragonés y Amérigo, 1998). Estas transformaciones se llevaron a cabo desde una perspectiva social y reconocían el potencial educativo de la experiencia estética (Alonso-Sanz, 
2017).

Se constató que el centro presentaba serios problemas en la calidad espacial y ambiental, que incluían un mal acondicionamiento térmico y acústico, problemas de seguridad, accesibilidad y salubridad, falta de organización de espacios, equipamiento y mobiliario en mal estado y una estética carcelaria, inadecuada para un centro escolar. Todas estas deficiencias se relacionan e interactúan con los núcleos de interés analizados, como podemos observar en la Figura 1. Se evidencia como el espacio condiciona aspectos fundamentales como la falta de interacción y convivencia del alumnado, la percepción del centro escolar tanto interna como externa, la inadecuada atención a la diversidad e inclusión del alumnado, la falta de diversidad de juego y actividades o la dificultad de llevar a cabo metodologías de aprendizaje más activas.

\section{Figura 1.}

Relación entre los aspectos educativos y sociales y las categorías relativas a la dimensión física del espacio.

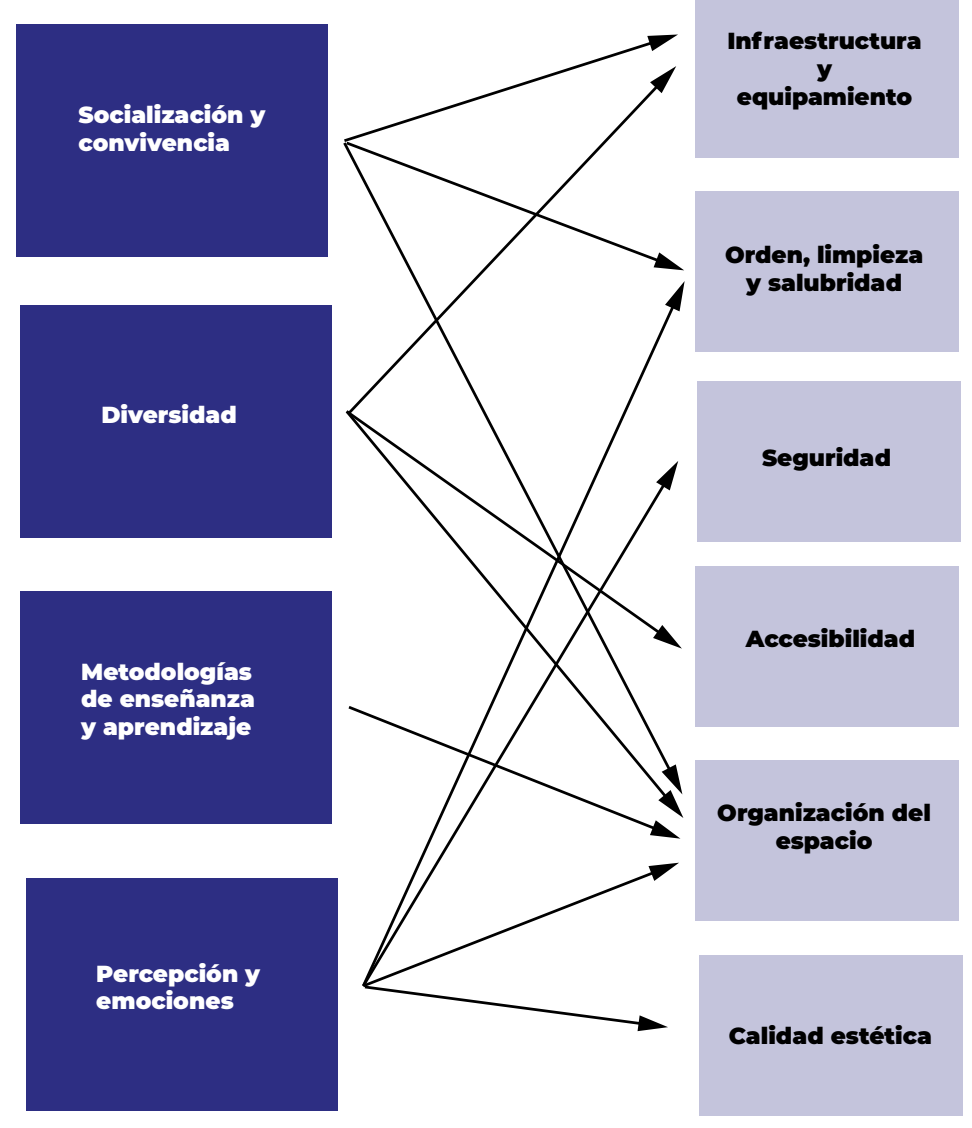


Las condiciones físicas espaciales conllevan barreras psicológicas y emocionales de toda la comunidad educativa que constituyen en sí mismas resistencias al cambio. La realidad física de un centro no puede ser un obstáculo para las decisiones de carácter organizativo y didáctico del profesorado (Crespo y Pino, 2007). De hecho, esta investigación ha constatado que aspectos como la implicación de personas de diversas disciplinas en el proyecto de transformación, entre otros factores, permite facilitar espacios de reflexión aportando nuevas posibilidades factibles de cambio.

Respecto a la metodología utilizada, distintas experiencias y estudios similares coinciden en la importancia de la multidisciplinariedad a lo largo del proceso, así como la participación de los distintos agentes de la comunidad educativa, en especial la del alumnado (González-Patiño et al., 2017; Valdés y Gutiérrez, 2018).

En la elaboración de este diagnóstico participativo como fase integrante de un proyecto IAP, se han utilizado distintas técnicas cualitativas y participativas que han permitido analizar el problema desde distintos vértices y contrastar resultados, a la vez que han contribuido a crear un clima de trabajo colaborativo. Otros estudios que han utilizado la combinación de técnicas evidencian la dificultad que esto requiere, pero el enriquecimiento de los resultados obtenidos (González-Patiño et al., 2017). Esta fase de investigación ha motivado y movilizado a la comunidad educativa, así como promovido el interés y los recursos de administraciones públicas, entidades sociales y colectivos.

Existe también coincidencia en señalar como factores que pueden obstaculizar la implementación de estos procesos la falta de recursos materiales e infraestructuras o de dotación económica (Valdés y Gutiérrez, 2018). Muchas experiencias han iniciado la puesta en marcha de estos proyectos sin tener en cuenta estos condicionantes, lo que ha repercutido en frustraciones y retrocesos. Por ello, uno de los aprendizajes de otras experiencias, en concreto la del proyecto de naturalización del patio del colegio Santos Samper en Almudévar, ha sido desarrollar acciones dirigidas a la movilización de recursos ya existentes en el propio centro educativo y en la 
comunidad, así como la búsqueda de recursos externos.

Siguiendo con las dificultades encontradas por otras investigaciones, se encuentran la falta de tiempo y la formación y experiencia por parte del profesorado en metodologías activas y participativas (Bolarin-Martínez y Yus, 2015; Paredes, 2004; Valdés y Gutiérrez, 2018). El profesorado de la escuela cuenta con una gran motivación y disposición por seguir formándose, lo que facilita el proceso.

Por último, algunas de las propuestas que se desprenden de este diagnóstico son la necesidad de formación y capacitación de la comunidad educativa, la provisión de espacios de participación y reflexión, la participación de todos los agentes implicados, así como la planificación y coordinación de las acciones futuras. Se propone crear ambientes de aprendizaje no solo en el aula sino en los distintos espacios del centro, promover la diversidad de juego y actividades, la flexibilidad y versatilidad del espacio, mejorar la calidad estética teniendo especialmente en cuenta aspectos como el orden y la organización, crear ambientes seguros y de cohesión, ampliar espacios abiertos a las familias y al barrio, entre otras. Es imprescindible continuar este proceso actuando sin precipitaciones, incluyendo procesos de evaluación y de generación de conocimientos en la línea metodología elegida, difundiendo las acciones desarrolladas y logros obtenidos en pos de la sostenibilidad y replicabilidad del trabajo realizado.

\section{Primeros pasos en la implementación del proyecto}

Una vez finalizada la fase de diagnóstico, se inició la puesta en marcha del proyecto participativo de transformación de espacios en el centro educativo. El primer paso fue la elección del nombre del proyecto, "Malvalima Rosalimón", y la toma de decisiones consensuada del plan de ejecución del mismo, las líneas de acción y la elección de las primeras medidas de transformación.

El proyecto comenzó su andadura, al inicio del curso 2019-2020, con una jornada de puertas abiertas (Figura 2) en la que se contó con la participación del conjunto de la comunidad educativa, así como de entidades sociales y colectivos de artistas. La finalidad era, por un lado, dar a conocer la realidad del centro y el pro- 
yecto, así como sensibilizar y motivar a los agentes implicados. La jornada se desarrolló durante todo el día y se realizaron varias actividades, entre las que destaca la realización de un mural colectivo en el patio del colegio (Figura 3), dando inicio a la transformación deseada. Cabe señalar la alta participación, especialmente de las familias y el alumnado, y el alto de grado de satisfacción del centro.

\section{Figuras 2 y 3.}

Jornada de puertas abiertas y elaboración del mural participativo.
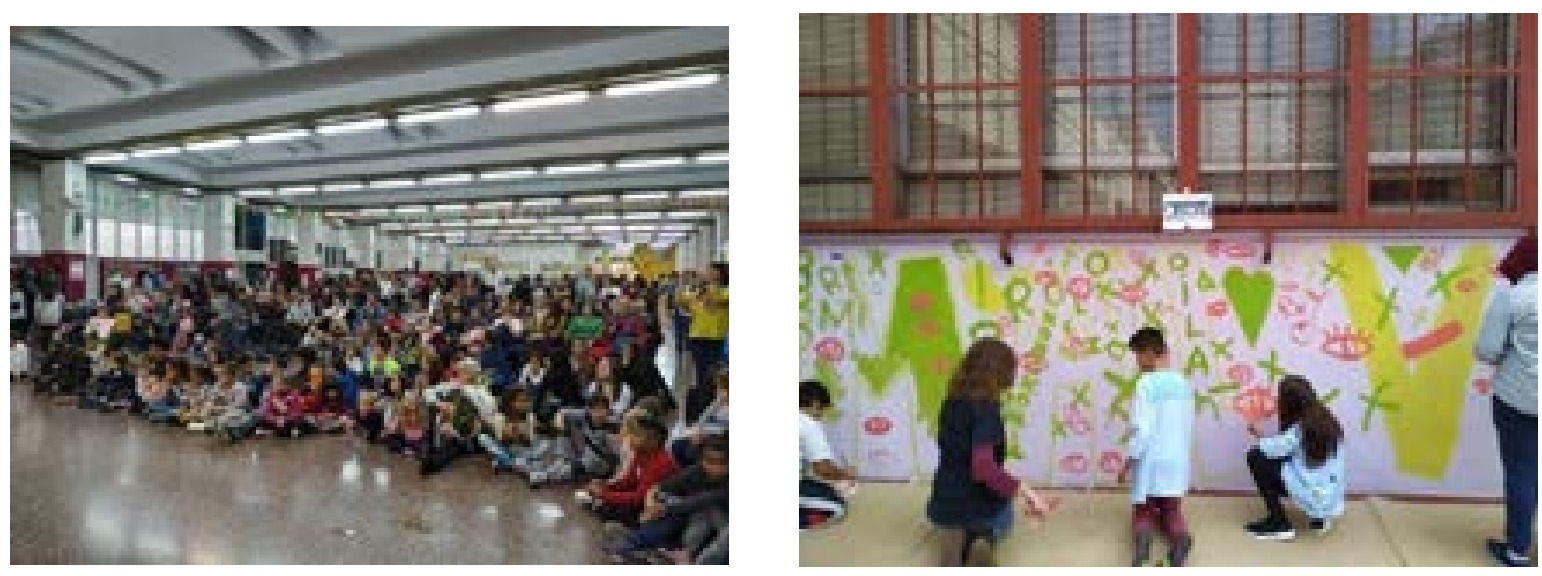

A lo largo de dicho curso académico, se llevaron a cabo acciones como pintar

la fachada exterior del centro con los colores que dan nombre al proyecto: malva, lima, rosa y limón, lo que cambió parte de la estética carcelaria del espacio exterior del colegio (Figura 4). A su vez, se crearon aulas temáticas como inglés y música, con distintos ambientes; así como espacios para actividades como yoga, un taller de reparación de bicicletas y una radio (Figura 5). Estas medidas están contribuyendo a visibilizar el centro educativo como un espacio abierto y dinámico dentro del barrio.

Además, se adecuó un espacio para las familias, donde estas pudieran reunirse y realizar actividades dirigidas para ellas, tales como costura e informática, lo que ha fomentado su participación en el centro. 


\section{Figuras 4 y 5.}

Fachada del patio exterior y taller de bicicletas.
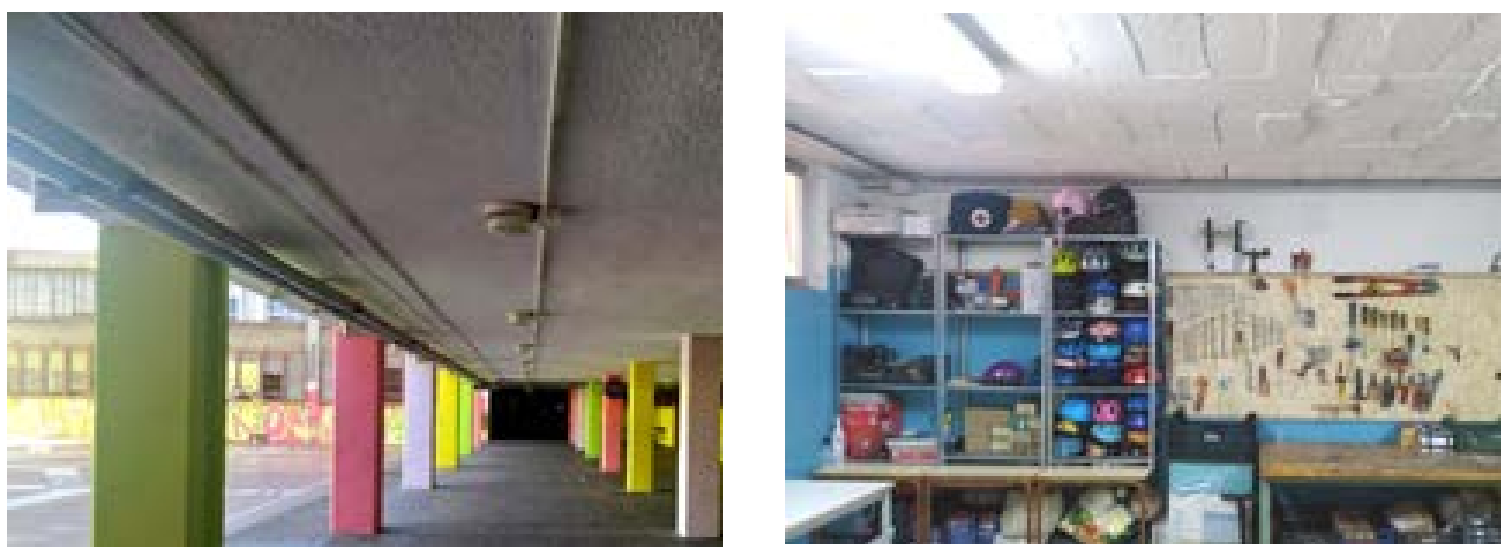

En el curso académico siguiente, 2020-2021, el proyecto ha continuado con la organización del profesorado en comisiones encargadas de cada uno de los espacios planteados para su transformación, limpieza y adecuación. A pesar de los pocos recursos que se disponen, se ha realizado la reforma de la biblioteca (Figura 6), gracias al trabajo incansable del equipo directivo y profesorado, y la ayuda de distintas entidades del barrio (PICH, CSL Ozanam de carpintería y CTL Cadeneta), así como de alumnos y alumnas de la Escuela de diseño de Zaragoza. También se ha continuado con la creación de aulas temáticas, como el aula científica, ligada al espacio exterior de huerto. Por último, con la ayuda de una entidad social, se han reformado los baños que se encontraban en peores condiciones.

\section{Figura 6.}

Antes y después del espacio de biblioteca.
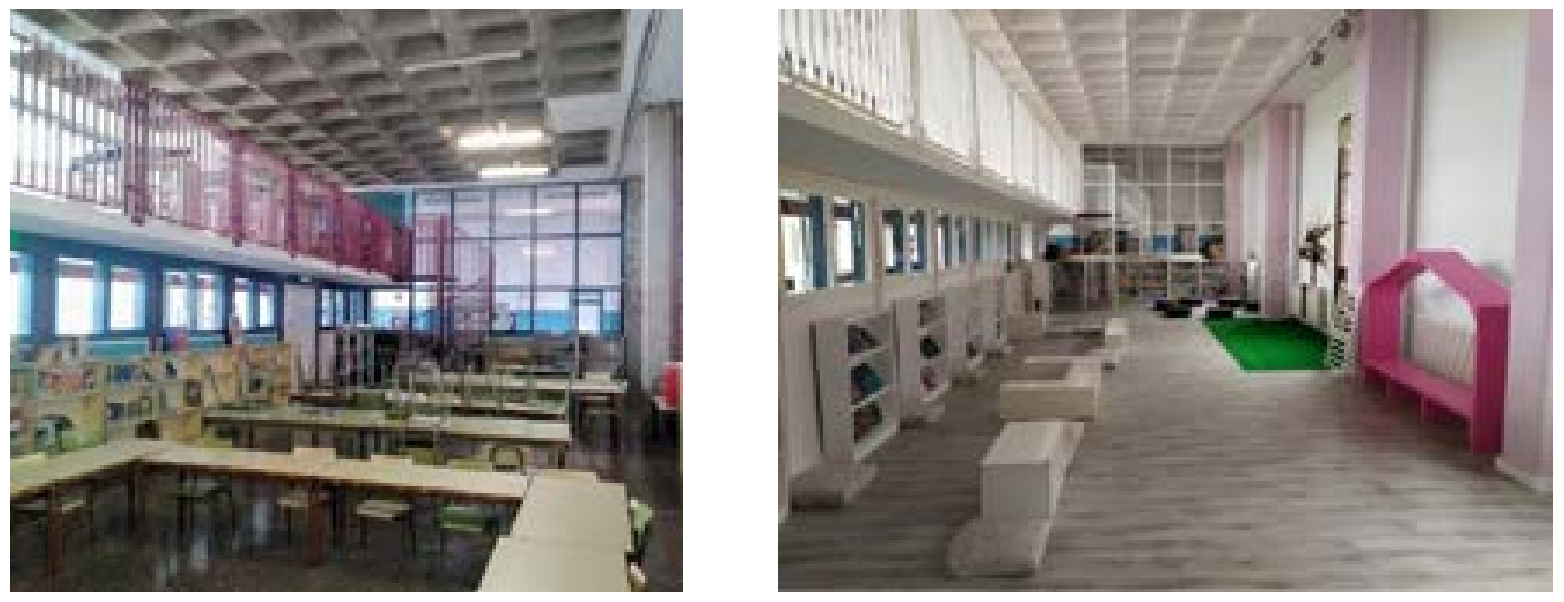
Hoy en día, el profesorado continúa dando forma al cambio metodológico de enseñanza, entendiendo el espacio del centro como un elemento indispensable. La falta de recursos y de apoyo institucional sigue siendo la mayor limitación del proyecto, así como la dificultad para realizar una investigación acción participativa sin un apoyo económico y logístico. Sin embargo, gracias la ilusión y el trabajo perseverante del equipo directivo y el claustro el proyecto continúa con los valores que le dieron inicio: la inclusión, la participación, los espacios colaborativos, las metodologías de enseñaza y aprendizaje activas, así como la idea de entender la escuela como un espacio abierto e integrado en su comunidad.

\section{REFERENCIAS}

Ainscow, M., Booth, T. \& Dyson, D. (2006). Improving school: Developing Inclusion. Routledge.

Aliaga, F. M., Gutiérrez-Braojos, C. y Fernández-Cano, A. (2018). Las revistas de investigación en educación: Análisis DAFO. Revista de Investigación Educativa, 36(2), 563-579. http://dx.doi.org/10.6018/rie.36.2.312461

Alonso-Sanz, A. (2017). Repensando tres entornos educativos consolidados. Artseduca, 18, 79-99. https://www.e-revistes.uji.es/index.php/artseduca/article/ view/2651/2181

Aragonés, J. I. y Amérigo, M. (1998). Psicología ambiental. Ediciones Pirámide.

Balcazar, F. E. (2003). Investigación acción participativa (IAP): Aspectos conceptuales y dificultades de implementación. Fundamentos en humanidades, 4(7-8), 59-77.

Balcazar, F. E., Suarez-Balcazar, Y. y Keys, C. B. (1998). Un modelo de investi-gación-acción para desarrollar la capacidad de comunidades para incrementar su poder. Suma Psicológica, 5, 123-147.

Barrett, P. S. y Zhang, Y. (2009). Optimal learning spaces: design implications for primary schools. SCRI Research Report. https://usir.salford.ac.uk/18471

Bautista, G. y Borges, F. (2013). Smart classrooms: Innovation in formal learning spaces to transform learning experiences. Bulletin of the Technical Committee 
on Learning Technology, 15 (3), 18-21.

Bazarra, L. y Casanova, O. (2016). La escuela ya no es un lugar. ArcixFormación.

Barba-Guamán, L., Valdiviezo-Diaz, P. y Aguilar, J. (2018). Gestión emergente de espacios colaborativos de aprendizaje. Revista Ibérica de Sistemas e Tecnologias de Informaçao, 4(15), 271-281. http://www.risti.xyz/issues/ristie15.pdf

Beach, D. y Dovemark, M. (2007). Education and the commodity problem: Ethnographic investigations of creativity and performativity in Swedish schools. The Tufnell Press.

Betrián, E.; Jové, G. y Farrero, M. (2014). El espacio total a través del arte. Revista nacional e internacional de investigación inclusiva, 7(1), 80-95.

Boddington, A. y Boys, J. (2011). Re-shaping Learning: A critical reader. The future of Learning Spaces in Post-Compulsory Education. Sense Publishers.

Bolarin-Martínez, M. J. y Yus, M. A. M. (2015). La coordinación docente en la universidad: retos y problemas a partir de Bolonia. Profesorado. Revista de Currículum y Formación de Profesorado, 19 (2), 319-332.

Cela, J., Fons, M. y Palou, J. (2016). Nuevos escenarios, nuevos aprendizajes. Dosier Graó, 7.

Cleveland, B. y Fisher, K. (2014). The evaluation of physical learning environments: a critical review of literature. Learning environments research, 17, 1-28. http:// dx.doi.org/10.1007/s10984-013-9149-3

Crespo, J. y Pino, M. R. (2007). Description of Environmental Factors in School. Internacional Review of Education, 53 (2), 205-218.

Delgado, V. (2009). Organización del aula de Educación Primaria en centros educativos de Burgos y su provincia. (Tesis de Licenciatura). Universidad de Burgos. Recuperado de Repositorio Institucional de la Universidad de Burgos

Errázuriz-Larraín, L. H. (2015). Calidad estética del entorno escolar: el (f)actor invisible. Arte, individuo y sociedad, 27(1), 87-100. http://dx.doi.org/10.5209/ rev_ARIS.2015.v27.n1.43861 
Fals, O. (1985). Conocimiento y poder popular. Siglo XXI.

González-Patiño, J., Esteban-Guitart, M. y San Gregorio, S. (2017). Participación Infantil en la Transformación de sus Espacios de Aprendizaje: Democratizando la Creación mediante un Proyecto de Fabricación Digital en un Fablab. Revista Internacional de Educación para la Justicia Social (RIEJS), 6(1), 137-154. http://dx.doi.org/10.15366/riejs2017.6.1.008

Hart, R. (2001). La participación de los niños en el desarrollo sostenible. PAU Education.

Kelz, C., Evans, G. W. \& Röderer, K. (2015). The Restorative Effects of Redesigning the Schoolyard: a Multi-Methodological, Quasi-Experimental Study in Rural Austrian Middle Schools. Environment and Behavior, 47, 119-139. http://dx. doi.org/10.1177/0013916513510528

Lewin, K. (1946). Action research and minority problems. Journal of Social Issues, 2, 34-46.

Martínez, M. (2000). La investigación-acción en el aula. Agenda académica, 7(1), 2739.

Novo, M. (2002). El derecho a conocer y el derecho a imaginar. En II Encuentro La ciudad de los niños: "Las transformaciones de la ciudad". Acción Educativa.

OECD (2013). Innovative learning environments. Educational Research and Innovation. OECD Publishing. http://dx.doi.org/10.1787/9789264203488-en

Paredes, J. (2004). Cultura escolar y resistencia al cambio. Tendencias pedagógicas, 9, 131-142.

PICH (2013). Plan Integral del Casco Histórico 2013-2020. Ayuntamiento de Zaragoza.

Pol, E. y Morales, M. (1986). El entorno escolar desde la Psicología Ambiental. En Jiménez, F. y Aragonés, J. I. (Comps.), Introducción a la Psicología Ambiental. Alianza Psicología.

Selener, D. (1997). Participatory action research and social change. Cornell University Participatory Action Research Network 
Staats, H. (2012). Restorative environments. En S. Clayton (Ed.), The Oxford handbook of environmental and conservation psychology (445-458). Oxford University Press.

Valdés, V. y Gutiérrez, P. (2018). COETUM. Proyecto para la creación de un espacio de ágora educativa. Evaluación de un proyecto de innovación para la transformación de la formación inicial del profesorado. Innovación educativa, (28), 189-204.

Vigo, B. y Soriano, J. (2014). Prácticas de enseñanza y aprendizaje creativo para una educación inclusiva en la formación inicial del profesorado. Experiencias de investigación. Revista nacional e internacional de investigación inclusiva, $7(1), 30-45$.

Viñao, A. (1993). El Espacio Escolar Introducción. Revista Historia de la educación, 12.

Wang, X. P., Zhang, J. y Yang, T. (2014). Hybrid SWOT approach for strategic planning and formulation in China worldwide express mail service. Journal of Applied Research and Technology, 12(2), 230-238. http://dx.doi.org/10.1016/ S1665-6423(14)72339-9

Fecha de recepción 12 de mayo de 2021 Fecha de aceptación 14 de noviembre de 2021

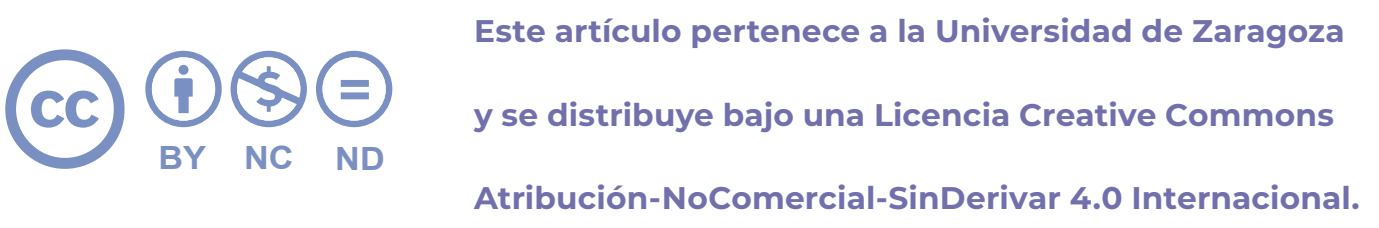

Eres libre de compartir copiar y redistribuir el material en cualquier medio o formato -

Bajo las condiciones siguientes:

- Reconocimiento de la autoría, ya incluida en esta diapositiva.

- NoComercial - no se puede utilizar el material para una finalidad comercial.

- SinObraDerivada - Sin remezclar, transformar o crear a partir del material. 